\title{
The expression of adhesion molecules in cigarette smoke-induced airways obstruction
}

\author{
S. González*, J. Hards, S. van Eeden+, J.C. Hogg ${ }^{+}$
}

\begin{abstract}
The expression of adhesion molecules in cigarette smoke-induced airways obstruction. S. González, J. Hards, S. van Eeden, J.C. Hogg. @ ERS Journals Ltd 1996.

ABSTRACT: Cigarette smoking produces peripheral airway inflammation in all smokers, and chronic airways obstruction in approximately $20 \%$ of heavy smokers. The present study was designed to test the hypothesis that airways obstruction is related to changes in the expression of adhesion molecules involved in the recruitment of cells to sites of inflammation in the lung.

Freshly resected lungs from heavy smokers with airways obstruction $(n=10)$ and from heavy smokers with normal lung function $(n=10)$ were collected in the operating room, inflated with optimal cutting temperature (OCT) medium and frozen over liquid nitrogen. Six micrometre thick cryostat sections cut from random samples of this tissue were stained, using immunohistochemistry, with monoclonal antibodies to the adhesion molecules on leucocytes: L-selectin, very late activation antigen-4 (VLA-4), CD11a/CD18, CD11b/CD18, CD11c/CD18; and on endothelial and epithelial surfaces: E-selectin, P-selectin, vascular cell adhesion molecule (VCAM-1), intercellular adhesion molecule (ICAM)-1 and ICAM-2 using the alkaline phosphatase anti-alkaline phosphatase (APAAP) technique. The slides were coded and the expression of each molecule scored by three observers using a semiquantitative grading system. Two inducible adhesion molecules, E-selectin on endothelium and CD11b on leucocytes, were also evaluated using quantitative morphometric analysis.

The results showed a distribution of adhesion molecules that was consistent with the inflammatory response in the airways and parenchyma of all subjects but failed to show any differences between those with or without airways obstruction.

We conclude that development of airways obstruction in heavy smokers cannot be explained by differences in the expression of adhesion molecules known to be involved in the control of cell traffic in the lung.

Eur Respir J., 1996, 9, 1995-2001.
\end{abstract}

*Dept of Pathology, School of Medicine, Universidad Catolica de Chile, Santiago de Chile, Chile. +Pulmonary Research Laboratory, University of British Columbia, St Paul's Hospital, Vancouver, BC, Canada.

Correspondence: J.C. Hogg

UBC Pulmonary Research Laboratory

St Paul's Hospital

1081 Burrard Street

Vancouver

BC V6Z 1Y6

Canada

Keywords: Adhesion molecules airways obstruction

lung inflammation

smoking

Received: February 131996

Accepted after revision July 141996

This study was supported by the Medical Research Council of Canada (Grant No. 4219).

Cigarette smoking induces a peripheral airways inflammatory response in all smokers [1], but several laboratories [2, 3], including our own [4], have shown that only a relatively small percentage of heavy smokers actually develop airways obstruction. The concept that this airways obstruction is due to an inflammatory process in the peripheral airways is well-established, but the mechanism by which the inflammatory response produces airways obstruction in certain people and not in others is unknown. The present study focuses on the expression of adhesion molecules that influence cell traffic in the peripheral lungs of smokers with airways obstruction.

The adhesion molecules that affect the migration of inflammatory cells out of the microvessels into the interstitium and airspaces of the lung have been categorized into the selectin, integrin and the immunoglobulin ( $\mathrm{Ig}$ ) families. The individual members of these three families have received a variety of names [5-9], and the terminology used in this study is based on LUSCINSKAS et al. [10]. The selectins include L-selectin (CD62L, lectin adhesion molecule-1 (LECAM-1), Mel-14, Leu8 , and leucocyte adhesion molecule-1 (LAM-1)), which is present on nearly all circulating leucocytes except a small subpopulation of T-cells, as well as E-selectin (endothelial leucocyte adhesion molecule-1 (ELAM-1), CD62E) and P-selectin (granulocyte-associated membrane protein-140 (GMP-140), platelet activation-dependent granule external membrane protein (PADGEM) and CD62P), which are inducible molecules on the endothelium. The integrins found on circulating leucocytes consist of the $\beta_{1}$-integrin, very late activation antigen4 (VLA-4) (CD49d/CD29 or $\left.\alpha_{4} \beta_{1}\right)$, and a group of three $\beta_{2}$-integrins which have a common $\beta$-chain (CD18) and three different $\alpha$-chains (CD11 a, b and c). The $\beta_{2}$-integrins CD11a/CD18 (leucocyte function-associated antigen-1 (LFA-1)), CD11b/CD18 (Mac-1, Mo1, complement receptor (CR)3) and CD11c/CD18 (p150, 95 and CR4) promote adherence of the leucocytes to intercellular adhesion molecule (ICAM)-1 (CD54) and ICAM-2 (CD $102)$, which are members of the Ig family present on the endothelium and epithelium. The $\beta_{1}$-integrin VLA-4 
on leucocytes interacts with the Ig family member vascular cell adhesion molecule-1 (VCAM-1) (CD106) on endothelial and interstitial cells.

Recent reports of studies on bronchial biopsies from normal nonsmokers, asymptomatic smokers and smokers with chronic bronchitis and airway obstruction suggest an increased expression of E-selectin in vessels and ICAM-1 on epithelial cells of patients with airways obstruction [11]. However, as this technique only provides tissue from the mucosa of airways that are central to the major site of airways obstruction, we decided to re-examine the problem using resected lung tissue, where all the airways are present. The present study was focused on the lungs of heavy smokers, to determine if there are differences in the expression of these adhesion molecules in heavy smokers who develop chronic airways obstruction compared to those who do not.

\section{Material and methods}

\section{Patient population}

The demographic and pulmonary function data from 20 patients whose lungs were examined in this study are summarized in table 1 . In all cases, this information was collected less than 1 week prior to surgery, using methods that have been described in detail previously [12]. The patients included 10 with airways obstruction and 10 with normal lung function, matched as closely as possible for age, sex and smoking history. All 20 were smokers, who required lung resections for small peripheral tumours.

The resected lung specimens were obtained immediately after excision and inflated with optimal cutting temperature cryo-embedding material (OCT TissueTek) diluted $50 \%$ with normal saline. The specimens were rapidly frozen over liquid nitrogen, cut into slices $2 \mathrm{~cm}$ thick using a band saw and randomly sampled with a power-driven hole saw. The samples were then stored at $-70^{\circ} \mathrm{C}$ until they were used.

Table 1. - Patient characteristics

\begin{tabular}{lcc}
\hline & Control & Obstructed \\
\hline Specimens examined $\mathrm{n}$ & 10 & 10 \\
Age yrs & $64 \pm 2$ & $67 \pm 3$ \\
Male \% & 80 & 80 \\
Pack-years & $43 \pm 6$ & $69 \pm 10$ \\
Chronic bronchitis n & 1 & 6 \\
FEV1 \% pred & $87 \pm 2$ & $57 \pm 3^{\dagger}$ \\
FEV1/FVC \% & $74 \pm 2$ & $55 \pm 2^{\dagger}$ \\
MMFR \% pred & $67 \pm 6$ & $28 \pm 2^{\dagger}$ \\
VC \% pred & $95 \pm 2$ & $87 \pm 4$ \\
TLC \% pred & $105 \pm 4$ & $123 \pm 7^{\ddagger}$ \\
RV \% pred & $114 \pm 12$ & $167 \pm 11^{\dagger}$ \\
TL,CO \% pred & $78 \pm 6$ & $71 \pm 6$ \\
PL,max \% pred & $89 \pm 15$ & $62 \pm 9$ \\
\hline
\end{tabular}

Values are presented as mean \pm SEM. FEV1: forced expiratory volume in one second; \% pred: percentage of predicted value; FVC: forced vital capacity; MMFR: maximum mid-expiratory flow rate; VC: vital capacity; TLC: total lung capacity; RV: residual volume; TL,CO: transfer factor of the lungs for carbon monoxide; $P$ L,max: maximum transpulmonary pressure. ${ }^{\dagger}$ : $\mathrm{p}<0.01 ;: \mathrm{p}<0.05$.

\section{Immunohistochemistry}

The immunohistochemical studies were performed on $6 \mu \mathrm{m}$ thick cryostat sections cut from each of these frozen cores of tissue. The sections were mounted onto glass slides coated with aminopropyltriethoxysilane (Silane). One section from each block was stained with haematoxylin and eosin for light microscopic examination and orientation. Immunostaining was then performed at room temperature using the alkaline phosphatase antialkaline phosphatase (APAAP) technique, according to Cordell et al. [13]. The antibodies chosen to detect them are listed in table 2. Briefly, the sections were air-dried and fixed in acetone for $10 \mathrm{~min}$. Nonspecific staining was blocked using 5\% normal rabbit serum in Tris-buffered saline (TBS), pH 7.6, for 15 min. One of a series of mouse monoclonal antibodies prepared in TBS containing $1 \%$ bovine serum albumin (TBS-BSA) (table 2) was applied to each slide for $30 \mathrm{~min}$ in a moist chamber. After washing twice in TBS, the sections were incubated with rabbit anti-mouse IgG (DAKOPATTS) in TBS-BSA, 1/20 dilution, for $30 \mathrm{~min}$. The TBS washings were repeated, followed by $30 \mathrm{~min}$ incubation with APAAP-complex (DAKOPATTS), 1/50 in TBS-BSA. After TBS washing, alkaline phosphatase substrate, containing new fuchsin as the capture agent and levamisole to block endogenous alkaline phosphatase, was applied for $20 \mathrm{~min}$. This gave a red colour at the site of the antigen-antibody reaction. After a final wash in TBS and tap water, the sections were counterstained with Mayer's haematoxylin and mounted with an aqueous mounting medium (Gelvatol).

Negative controls were prepared by omitting the primary antibody and by replacing the primary antibody with mouse nonimmune IgG (Sigma).

\section{Assessment of staining}

Two methods for assessing the presence and intensity of staining of the various adhesion molecules were used. Firstly, a semi-quantitative scoring system was designed to evaluate the pattern and intensity of staining for each monoclonal antibody. This system allowed the staining to be graded as either negative (grade

Table 2. - Monoclonal antibodies, sources and working concentrations

\begin{tabular}{llc}
\hline MoAb & Source & $\begin{array}{c}\text { Working } \\
\text { concentration } \\
\mu \mathrm{g} \cdot \mathrm{mL}^{-1}\end{array}$ \\
\hline E-selectin & R \& D Systems & 0.1 \\
P-selectin & Serotec & 1.0 \\
L-selectin & Becton Dickinson & 0.5 \\
VLA-4 & AMAC Inc. & 0.4 \\
CD11a & DAKO & 0.2 \\
CD11b & DAKO & 0.2 \\
CD11c & DAKO & 0.1 \\
CD18 & DAKO & 0.2 \\
VCAM-1 & R \& D Systems & 2.0 \\
ICAM-2 & Courtesy of T.A. Springer & 2.5 \\
ICAM-1 & AMAC Inc. & 0.4
\end{tabular}

MoAb: monoclonal antibody; VLA-4: very late activation antigen-4; VCAM-1: vascular cell adhesion molecule-1; ICAM: intercellular adhesion molecule-1. 
$0:-$ ), weakly positive (grade $1:+$ ), moderately positive (grade $2:++$ ) and intensely positive (grade 3: +++ ). An average grading score was allocated for each slide, after evaluating the entire slide. Slides were evaluated by three observers and all tissue was examined without knowledge of the patient's functional status. The staining was evaluated separately in the following areas: the pulmonary and bronchial blood vessels, including capillaries, venules, arterioles, and larger vessels (diameter $>100 \mu \mathrm{m})$; the alveolar and bronchial epithelia; leucocytes, including the alveolar macrophages; the extravascular lymphoid cells (bronchus-associated lymphoid tissue (BALT)) and intravascular and extravascular leucocytes. Intra- and interobserver variability of the grading system were determined between the three observers, and by one observer evaluating the slides 3 weeks apart.

Secondly, standard morphometric methods were used to quantify the expression of two adhesion molecules [14]. For this part of the study, we measured the presence and intensity of staining of two adhesion molecules, CD11b on leucocytes and E-selectin on endothelial surfaces, that are known to upregulate during inflammation. For CD11b evaluation, a point counting technique was applied [15] using a Nikon microscope, which allowed random fields of view generated by a computer program to be analysed at $\times 800$ magnification. A point counting grid of 200 points was superimposed on to the microscope image, a total of 10 fields was counted on each slide, and the volume fraction $(\mathrm{Vv})$ of leucocytes was calculated from the equation:

$$
\left.V_{V} \text { (leucocytes }\right)=\frac{\sum \text { points on leucocytes }}{\sum \text { of all points }}
$$

The volume fraction of either positive or negative leucocytes was expressed as a fraction of the anatomical structure where the leucocytes were located, for example in alveolar walls:

$$
\mathrm{Vv} \text { (granulocytespos) }=\frac{\sum \text { of points on granulocytespos }}{\sum \text { of all points on alveolar walls }}
$$

Similar calculations were made for negative granulocytes and for leucocytes in the airspace.

For E-selectin, the staining was assessed using a computerized high resolution true colour image analysis system (Bioviews Infra-scan $®$ ). This system determines the intensity, saturation and the hue of a colour in a digitized field of view. To determine the E-selectin expression on pulmonary capillary endothelium, for example, we selected 10 random fields of view from each slide, point counted to determine the fraction of alveolar wall, and then used the segmenting function and colour thresholding to assess the fraction of positively stained pulmonary capillary endothelium in alveolar wall. For estimating the amount of staining on the endothelial surface of the large blood vessels, the area of the vessel wall was digitized, and the fraction of positive staining in the area was computed using colour thresholds. A similar method was employed to estimate the amount of positive staining in the bronchial vessels of larger airways.

\section{Statistical analysis}

Statistical analysis was performed using SYSTAT version 5.1 software (Systat Inc., Evanston, IL, USA). Students t-tests were used to compare differences in the volume fractions of leucocytes and surface areas of tissue between the obstructed and the nonobstructed groups. The sequential rejective Bonferroni procedure was used to correct for multiple comparisons. Values are expressed as the mean \pm SEM and considered significant if $p$ value was less than 0.05 .

The inter- and intraobserver variation of the grading system were evaluated by calculating the Pearson coefficient of mean-square contingency $\left(R_{2}\right)$ for each grade and expressing $R_{2}$ as a fraction of the maximum possible value, $R_{2 \max }$ which represents the value of the Pearson Chi-squared coefficient if the there is $100 \%$ agreement in the grading score between or within observers: thus, $\mathrm{R}_{2 \max }= \pm 0.82$ [15].

\section{Results}

Figure 1 shows examples of the staining obtained for the different adhesion molecules. E-selectin (fig. 1a) is shown on the endothelial surface within an alveolar wall, P-selectin (fig. 1b) on the endothelium in the bronchial circulation, and staining for L-selectin (fig. 1c) on lymphocytes in BALT. Staining of the $\beta_{1}$-integrin VLA4 (fig. 1d) is also shown on lymphocytes in BALT, and staining for the $\beta_{2}$-integrin CD11c (fig. 1e) on an alveolar macrophage. Results obtained with antibodies against the Ig family show staining for VCAM-1 on the endothelium of a pulmonary arteriole (fig. 1f), staining for ICAM-2 on a bronchial vessel (fig. 1g), and staining for ICAM-1 on the alveolar epithelium (fig. 1h).

\section{Qualitative analysis}

When the semiquantitative analysis of adhesion molecule expression was analysed to assess whether there were any differences between the staining reactions of lungs from smokers with normal lung function compared with lungs from smokers with airways obstruction, none were found for any of the antibodies tested. Tables 3 and 4 summarize the intensity and the distribution of the staining on the endothelia of the pulmonary and bronchial circulations, the epithelia of the conducting airways and alveolar surfaces, and the migrating inflammatory cells both for the obstructed (table 3 ) and nonobstructed (table 4) cases.

The antibodies to E- and P-selectin, VCAM-1, ICAM1 and ICAM-2, consistently stained the endothelia of the pulmonary circulation with moderate to strong intensity. They also stained the endothelia of the bronchial circulation, except in patients without airway obstruction, where the arterioles/venules and larger vessels showed no staining for ICAM-1 and ICAM-2.

The alveolar epithelium showed intense and continuous staining for ICAM-1, but no staining for any other adhesion molecule. This staining for ICAM-1 was also concentrated on epithelial cells that were participating in the repair process, particularly those that appeared to be in transition between Type II and Type I cells.

Interestingly, anti-ICAM-1 and anti-ICAM-2 produced weak staining of the alveolar macrophages. In contrast, 

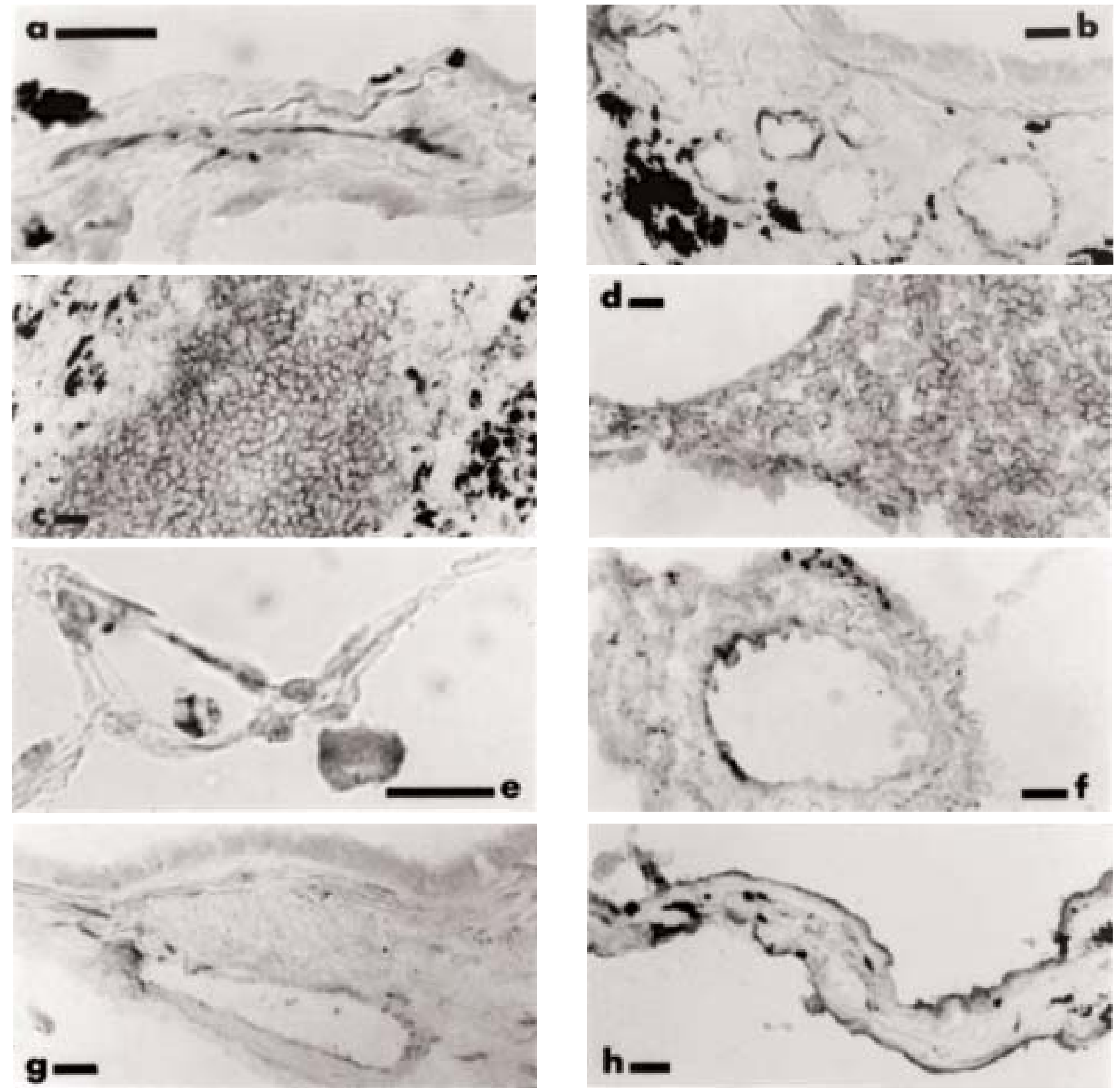

Fig. 1. - Light micrographs of human lung tissue showing staining for the following adhesion molecules: a) E-selectin on pulmonary microvessels; b) P-selectin on bronchial vessels in an airway wall; c) L-selectin on lymphocytes in BALT; d) VLA-4 on lymphocytes in BALT; e) CD11c on alveolar macrophages; f) VCAM-1 on a pulmonary arterioles; g) ICAM-2 on a bronchial vessel in an airway wall; and h) ICAM-1 on alveolar epithelium. BALT: bronchus-associated lymphoid tissue; VLA-4: very late activation antigen-4; VCAM-1: vascular cell adhesion molecule1; ICAM: intercellular adhesion molecule. (Internal scale bar $=20 \mu \mathrm{m}$ ).

the CD11a, CD11b, and CD11c/CD18 antibodies produced moderate to strong staining. Alveolar macrophages also stained weakly for the L-selectin and VLA-4.

Lymphocytes in the extravascular region, BALT, stained positively for the integrins and for L-selectin. BALT also stained with VCAM-1. They showed weak staining for ICAM-1.

Intense staining of the granulocytes in the lung tissue was observed with all the integrins and with L-selectin. The granulocytes observed in alveoli and bronchi were negative for L-selectin, which is consistent with shedding of L-selectin during the migration process. Moderate staining was observed with ICAM-1 and ICAM-2.

The integrin VLA-4 produced variable staining of alveolar macrophages, strong staining of lymphocytes in the BALT and also a population of granulocytes. The majority of these VLA-4 positive granulocytes were eosinophils, as defined by additional studies using a double staining technique identifying eosinophils with eosinophil specific peroxidase, the rest being activated neutrophils [16].

The $R_{2 \max }$ value comparing different observers ranged from 0.527 (lymphocytes) to 0.735 (pulmonary microvessels). Although this shows some variation between observers, analysing each observer's results separately showed that they all came to the same conclusion. Intraobserver variability was better $\left(\mathrm{R}_{2 \max }=0.735\right)$ and results reported in tables 3 and 4 are from a single observer. 
Table 3. - Staining of adhesion molecules in patients with airway obstruction

\begin{tabular}{|c|c|c|c|c|c|c|c|c|}
\hline & E-selectin & P-selectin & L-selectin & VLA-4 & CD11* & VCAM-1 & ICAM-2 & ICAM-1 \\
\hline \multicolumn{9}{|l|}{ Pulmonary circulation } \\
\hline Microvessels & + & ++ & - & - & - & + & ++ & +++ \\
\hline Arterioles & ++ & ++ & - & - & - & ++ & ++ & ++ \\
\hline Larger vessels (diam. >100 $\mu \mathrm{m})$ & + & ++ & - & - & - & ++ & + & ++ \\
\hline \multicolumn{9}{|l|}{ Bronchial circulation } \\
\hline Capillaries & +++ & +++ & - & - & - & ++ & ++ & + \\
\hline Arterioles/venules & ++ & +++ & - & - & - & ++ & ++ & + \\
\hline \multirow{2}{*}{\multicolumn{9}{|c|}{ Surface epithelium }} \\
\hline & & & & & & & & \\
\hline Bronchial epithelium & - & - & - & - & - & - & - & ++ \\
\hline Alveolar epithelium & - & - & - & - & - & - & - & ++ \\
\hline \multicolumn{9}{|l|}{ Inflammatory cells } \\
\hline Alveolar macrophages & - & - & + & + & +++ & - & + & ++ \\
\hline Carbon pigment-laden macrophages & - & - & - & - & ++ & - & - & + \\
\hline Haemosiderin-laden macrophages & - & - & - & - & +++ & - & - & + \\
\hline Extravascular lymphocytes & - & - & +++ & +++ & ++ & ++ & - & + \\
\hline Granulocytes & + & - & +++ & +++ & +++ & - & ++ & ++ \\
\hline
\end{tabular}

*: this group includes monoclonal antibodies (MoAbs) against CD11a, CD11b, CD11c and CD18 (the staining was similar for all of them). Grading system: +: weakly positive; ++: moderately positive; +++: intensely positive; -: negative. VLA-4: very late activation antigen-4; VCAM-1: vascular cell adhesion molecule-1; ICAM: intercellular adhesion molecule.

Table 4. - Staining of adhesion molecules in patients without airway obstruction

\begin{tabular}{|c|c|c|c|c|c|c|c|c|}
\hline & E-selectin & P-selectin & L-selectin & VLA-4 & CD11* & VCAM-1 & ICAM-2 & ICAM-1 \\
\hline \multicolumn{9}{|l|}{ Pulmonary circulation } \\
\hline Microvessels & + & ++ & - & - & - & + & ++ & +++ \\
\hline Arterioles & + & ++ & - & - & - & ++ & ++ & ++ \\
\hline \multirow{2}{*}{\multicolumn{9}{|c|}{ Bronchial circulation }} \\
\hline & & & & & & & & \\
\hline Capillaries & ++ & +++ & - & - & - & ++ & ++ & + \\
\hline Arterioles/venules & ++ & +++ & - & - & - & ++ & - & - \\
\hline Larger vessels (diam. $>100 \mu \mathrm{m})$ & + & ++ & - & - & - & ++ & - & - \\
\hline \multicolumn{9}{|l|}{ Surface epithelium } \\
\hline Bronchial epithelium & - & - & - & - & - & - & - & + \\
\hline Alveolar epithelium & - & - & - & - & - & - & - & +++ \\
\hline \multicolumn{9}{|l|}{ Inflammatory cells } \\
\hline Alveolar macrophages & - & - & + & + & +++ & - & + & + \\
\hline Carbon pigment-laden macrophages & - & - & - & - & ++ & - & - & + \\
\hline Haemosiderin-laden macrophages & - & - & - & - & +++ & - & - & + \\
\hline Extravascular lymphocytes & - & - & +++ & +++ & ++ & +++ & - & + \\
\hline Granulocytes & + & - & +++ & +++ & +++ & - & ++ & ++ \\
\hline
\end{tabular}

*: this group includes MoAbs against CD11a, CD11b, CD11c and CD18 (the staining was similar for all of them). For grading system and definitions see legend to table 3. Differences in scores between table 3 and table 4 were not significantly different.

\section{Quantitative analysis}

Quantitative analysis of CD11b (fig. 2) and E-selectin (fig. 3) showed no difference in the expression of these two adhesion molecules in lungs of smokers with and without airways obstruction. This supports the findings of the qualitative evaluation of the tissue. In the quantitative analysis of CD11b, leucocytes were categorized as either positive or negative. The majority of both alveolar macrophages and polymorphonuclear neutrophils (PMNs) in alveolar walls or spaces stained positive for CD11b, with the majority of lymphocytes in the alveolar walls staining negative. Figures $2 \mathrm{a}$ and $\mathrm{b}$ shows the PMNs in the alveolar walls and alveolar macrophages in the alveolar spaces, with no differences between the two groups. Both bronchial vessels in airway walls and the endothelium of pulmonary vessels stained strongly for E-selectin, but quantifying the area and the intensity of staining gave similar results in both groups (fig. 3). Staining of E-selectin on pulmonary capillaries in alveolar walls was less intense than on larger vessels, but similar in both groups.

\section{Discussion}

The early studies of NIEwOEHNER et al. [1] established that cigarette smoking produces an inflammatory response in the peripheral lung of all who smoke. This implicated the inflammatory process in the pathogenesis of smoking-induced chronic obstructive pulmonary disease (COPD), and led to the current working hypothesis that the inflammatory process is amplified by host and/or environmental factors to narrow the lumen of the airways in the $20 \%$ of smokers that actually develop airways obstruction [2-4]. A previous report has suggested an increased expression of ICAM-1 and E-selectin in bronchial biopsies from patients with obstructive 

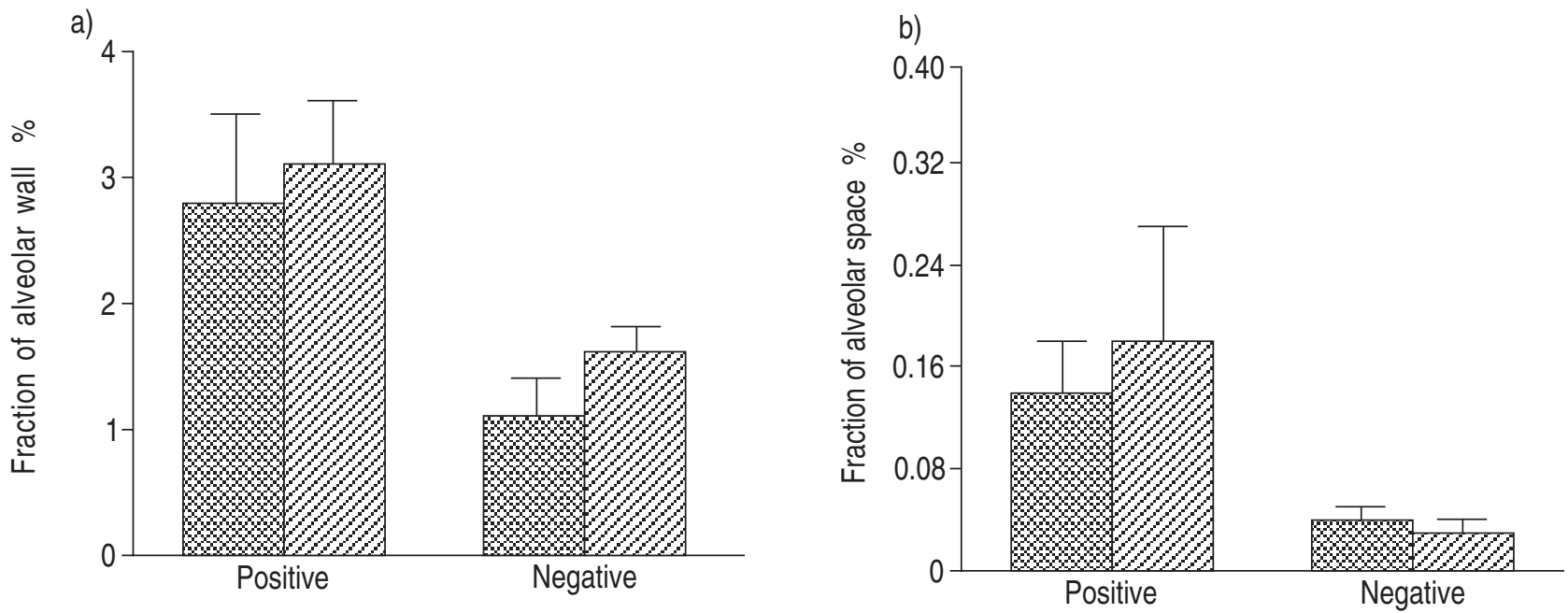

Fig. 2. - The expression of CD11b: a) on granulocytes in alveolar walls; and b) on alveolar macrophages in alveolar spaces in smokers with $(\mathrm{n}=5)$ and without $(\mathrm{n}=5)$ airways obstruction. Using quantitative immunohistochemistry (see Methods section) CD11b positive and negative leucocytes were expressed as a fraction of alveolar wall or alveolar space. Values are presented as mean \pm sem. No significant difference was seen

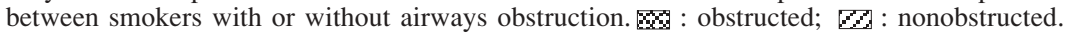

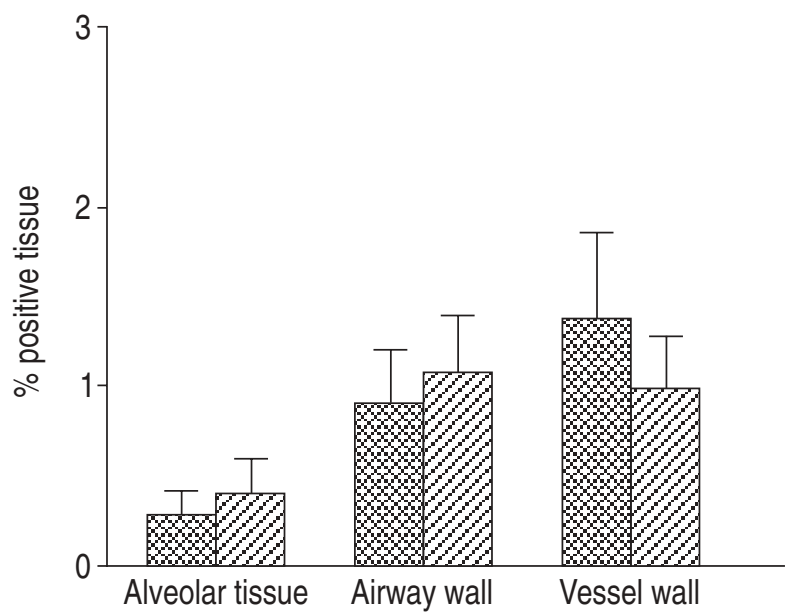

Fig. 3. - The expression of E-selectin on vascular endothelium of airway walls, larger pulmonary vessels and pulmonary microvessels. Quantitative immunohistochemistry was used and the staining for Eselectin is expressed as the fraction of a digitized airway, vessel or alveolar wall in 10 randomly selected fields. Values are presented as mean \pm SEM. No significant difference was observed between groups.

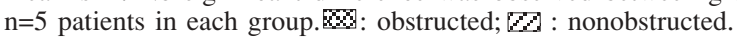

bronchiolitis [11]. This report established clear differences between smokers and nonsmokers and less marked differences between smokers with or without airways obstruction. Our study concentrated on the two latter groups and on the peripheral airways, which are the major site of airways obstruction. The data provide no evidence that an overexpression of any adhesion molecule is associated with the inflammatory events responsible for airways obstruction.

The vascular endothelial cell adhesion molecules Eand P-selectin, and the Ig superfamily (ICAM-1, ICAM2 and VCAM-1) were all moderately expressed both on the pulmonary and bronchial vascular endothelium. E- and P-selectin and ICAM-1 are inducible molecules, which are present in low copy number in normal lung tissue, and their moderate expression in the vascular endothelia suggests that there is a permanent and intense activation of the endothelium by inflammatory mediators in lung tissue of heavy smokers. Leucocytes need the selectin group of adhesion molecules to tether them onto activated endothelium in postcapillary venules in the systemic circulation [17]. This is followed by firm adhesion via the CD11/CD18 on leucocytes to the ICAMs on endothelium, preparing the cell for emigration. In the pulmonary microvessels, this initial tethering step is unnecessary because leucocytes are trapped in pulmonary capillaries due to their relatively larger size compared to pulmonary capillary segments [18]. We discerned no clear differences in the expression of the endothelial-associated selectins between pulmonary and bronchial vessels in the present studies. Triggering of leucocytes through the selectins has recently been suggested as an important function of this group of molecules [19]. This triggering function may be important in the recruitment of leucocytes in the pulmonary microvessels.

Both the alveolar and bronchial epithelial surfaces stained only with anti-ICAM-1. The staining of alveolar epithelium seemed to be most intense in areas where there was evidence of an alveolar repair process with Type II cell hyperplasia. The unique location of this molecule on the alveolar surface has been reported previously [20], and suggests that it could be important in controlling the movement of leucocytes onto and over the alveolar surface.

The strong staining of certain granulocytes with antiVLA-4 is of interest. Neutrophils do not express this adhesion molecule [21, 22], and we observed that the strongly staining PMNs in the present study were mostly eosinophils. It was hypothesized that pre-existing airway hyperresponsiveness was an important host factor that predicted the development of COPD [23]. In an animal model of allergic airways hyperresponsiveness, partly due to eosinophils, inflammation can be blocked by antibodies to ICAM-1 [24]. However, the present study failed to demonstrate any difference in the expression of ICAM-1 on the endothelium in the lung tissue of the two groups. The mononuclear cells that migrated into the inflammatory response stained weakly $(+)$ for proteins normally found on the endothelium. 
For example, the macrophages stained for ICAM-1 and ICAM-2. Moreover, members of the lymphocyte population stained for ICAM-1 and VCAM-1. It is currently accepted that CD11a/CD18 and CD11b/CD18 form ligand receptor pairs with the ICAM-1 as the leucocyte adheres to and crosses the endothelium [25]. We suspect that both ICAM-1 and VCAM-1 may be retained by the lymphocytes by remaining with their respective ligands CD11/18 and VLA-4 on the cell surface after the lymphocytes have moved out of the vascular space and into the interstitial tissue. Alternatively, this promiscuous receptor could also be expressed in low copy number on leucocytes to help with leucocyte aggregation [26].

E-selectin on endothelium and CD11b on leucocytes, the two adhesion molecules selected for quantitative analysis, are both pivotal adhesion molecules in the recruitment of leucocytes to sites of inflammation. The molecules are both normally expressed in low copy numbers, and E-selectin is rapidly induced by inflammatory cytokines, such as tumour necrosis factor (TNF) and interleukin (IL)-1, and CD11b by IL-8 [9]. The moderate staining of E-selectin both in pulmonary and bronchial vascular endothelium is consistent with inflammatory stimulation of both of these vascular beds. However, no significant differences were found either in the pattern or the intensity of E-selectin expression on pulmonary and bronchial vascular endothelium between the obstructed and non-obstructed smokers. Quantitative analysis of CD11b on granulocytes showed results that were consistent with the qualitative observations.

In summary, the results reported here suggest that the expression of the adhesion molecules in the peripheral lung of cigarette smokers is consistent with the inflammatory reaction induced by smoking. However, there were no differences in their expression to indicate that these molecules have any particular role in the pathogenesis of the structural changes in the airways responsible for the obstruction that occurs in a proportion of the smoking population.

Acknowledgements: The authors would like to thank S. Greene for photographic assistance, L. Verburgt for the statistical analysis and K. Webb for help in preparation of this manuscript.

\section{References}

1. Niewoehner DE, Kleinerman J, Rice DB. Pathological changes in the peripheral airways of young cigarette smokers. N Engl J Med 1974; 291: 755-758.

2. Fletcher $\mathrm{C}$, Peto R. The natural history of chronic airway obstruction. Br Med J 1977; 1: 1645-1648.

3. Lebowitz MD, Knudson RJ, Burrows B. Tucson epidemiological study of obstructive lung disease. 1. Methodology and prevalence of disease. Am J Epidemiol 1977; 102: 137-152.

4. Wright JL, Lawson LM, Paré PD, Kennedy S, Wiggs B, Hogg, JC. The detection of small airways disease. Am Rev Respir Dis 1984; 129: 989-994.

5. Springer TA. Adhesion receptors of the immune system. Nature 1990; 346: 425-434.

6. Albelda SM. Endothelial and epithelial adhesion molecules. Am J Respir Cell Mol Biol 1991; 4: 195-203.
7. Montefort S, Holgate ST. Adhesion molecules and their role in inflammation. Respir Med 1991; 85: 91-99.

8. Carlos TM, Harlan JM. Membrane proteins involved in phagocyte adherence to endothelium. Immunol Rev 1990; 114: 5-28.

9. Carlos TM, Harlan JM. Leukocyte-endothelial adhesion molecules. Blood 1994; 84: 2068-2101.

10. Luscinskas FW, Lawler J. Intergrins as dynamic regulators of vascular function. FASEB J 1994; 8: 929-938.

11. Di Stefano A, Maestrelli P, Roggeri A, et al. Upregulation of adhesion molecules in the bronchial mucosa of subjects with chronic obstructive bronchitis. Am J Respir Crit Care Med 1994; 149: 803-810.

12. Bosken CH, Hards J, Gatter K, Hogg JC. Characterization of the inflammatory reaction in the peripheral airways of cigarette smokers using immunocytochemistry. Am Rev Respir Dis 1992; 145: 911-917.

13. Cordell JL, Falini B, Erber WN, et al. Immunoenzymatic labelling of monoclonal antibodies using immune complexes of alkaline phosphatase and monoclonal antialkaline phosphatase (APAAP complexes). J Histochem Cytochem 1984; 32: 219-229.

14. Cruz-Orive LM, Weibel ER. Sampling designs for stereology. J Microscopy 1987; 122: 235-257.

15. Sachs L. Applied Statistics. A Handbook of Techniques. New York, NY, Springer Verlag, 1992; p. 537.

16. Johnson $\mathrm{B}$, Issekutz $\mathrm{T}$, Kubes $\mathrm{P}$. The $\alpha_{4}$-integrin supports leukocyte rolling and adhesion in vivo. FASEB J 1996; 10: A612.

17. Von Adrian UH, Hansell P, Chambers JD, et al. Lselectin is required for $\beta_{2}$-integrin-mediated neutrophil adhesion at physiological shear rate in vivo. Am J Pathol 1992; 263: H1034-H1044.

18. Doerschuk CM, Beyers N, Coxson HO, Wiggs BR, Hogg JC. The importance of neutrophil and capillary diameter in the margination of PMN in the lung. $J$ Appl Physiol 1993; 74: 3040-3045.

19. Crockett-Torabi E, Sulenbarger B, Smith WC, Fantone JC. Activation of human neutrophils through L-selectin and Mac-1 molecules. J Immunol 1995; 154: 2298-2302.

20. Burns AR, Takei F, Doerschuk CM. Quantitation of ICAM-1 expression in mouse lung during pneumonia. J Immun 1994; 153: 3189-3194.

21. Walsh GM, Mermod J, Hartnell A, Kay AB, Wardlaw AJ. Human eosinophil, but not neutrophil, adherence to IL-1-stimulated human umbilical vascular endothelial cells is very late antigen-4 dependent. J Immunol 1991; 146: 3419-3423.

22. Schleimer RP, Sterbinsky SA, Kaiser J, et al. IL-4 induces adherence of human eosinophils and basophils but not neutrophils to endothelium. J Immunol 1992; 148: 1086-1092.

23. Orie NG, Sluiter HJ, DeVries K. The host factor in bronchitis. In: Orie NG, Sluiter HJ, eds. Bronchitis. Assen, Royal VanGorcum, 1961; pp. 43-59.

24. Wegner CD, Gundel RH, Reilly P, Haynes N, Letts LG, Rathlein R. Intercellular adhesion molecule-1 (ICAM1) in the pathogenesis of asthma. Science 1990; 247: 456-459.

25. Marlin SD, Springer TA. Purified intercellular adhesion molecule-1 is ligand for lymphocyte function-associated antigen-1. Cell 1987; 51: 813-819.

26. Kuypers T, Koendermank W, Weening RS, Verhoeven AJ, Roos D. Continuous cell activation is necessary for the stable interaction of complement receptor 3 with its counter structure in the aggregation of human neutrophils. Blood 1989; 20: 501-507. 